\title{
Characterization of epitaxial silicene with Raman spectroscopy
}

DOI:

10.1103/PhysRevB.98.075437

\section{Document Version}

Final published version

Link to publication record in Manchester Research Explorer

\section{Citation for published version (APA):}

Kukucska, G., Zólyomi, V., \& Koltai, J. (2018). Characterization of epitaxial silicene with Raman spectroscopy. Physical Review B, 98(7), [075437]. https://doi.org/10.1103/PhysRevB.98.075437

\section{Published in:}

Physical Review B

\section{Citing this paper}

Please note that where the full-text provided on Manchester Research Explorer is the Author Accepted Manuscript or Proof version this may differ from the final Published version. If citing, it is advised that you check and use the publisher's definitive version.

\section{General rights}

Copyright and moral rights for the publications made accessible in the Research Explorer are retained by the authors and/or other copyright owners and it is a condition of accessing publications that users recognise and abide by the legal requirements associated with these rights.

\section{Takedown policy}

If you believe that this document breaches copyright please refer to the University of Manchester's Takedown Procedures [http://man.ac.uk/04Y6Bo] or contact uml.scholarlycommunications@manchester.ac.uk providing relevant details, so we can investigate your claim.

\section{OPEN ACCESS}




\title{
Characterization of epitaxial silicene with Raman spectroscopy
}

\author{
G. Kukucska, ${ }^{1}$ V. Zólyomi, ${ }^{2}$ and J. Koltai ${ }^{1}$ \\ ${ }^{1}$ Department of Biological Physics, Eötvös Loránd University, Pázmány Péter sétány 1/A, 1117 Budapest, Hungary \\ ${ }^{2}$ National Graphene Institute, University of Manchester, Oxford Road, Manchester M13 9PL, United Kingdom
}

(Received 27 November 2017; revised manuscript received 18 June 2018; published 30 August 2018)

\begin{abstract}
Silicene, the silicon equivalent of graphene, is most commonly grown on $\mathrm{Ag}(111)$ substrates where it undergoes reconstruction due to the strong interaction between the $\mathrm{Si}$ and $\mathrm{Ag}$ atoms. We demonstrate through first-principles density functional theory for eight reconstructions that the Raman spectrum is unique for each configuration. We argue that the reconstructions can, in fact, be identified by their Raman spectra and suggest key features within the spectra as points of reference to be used for identification.
\end{abstract}

DOI: 10.1103/PhysRevB.98.075437

\section{INTRODUCTION}

Silicene is a two-dimensional (2D) honeycomb lattice of silicon atoms which, unlike its carbon counterpart graphene [1], exhibits a sublattice buckling, that is, the $A$ and $B$ sublattices of the honeycomb structure are vertically shifted relative to one another (see Fig. 1). While predicted to be stable by density functional theory in 1994 [2], its synthesis was not possible until the early 2010s [3-6]. Silicene is typically grown by depositing $\mathrm{Si}$ atoms from a preheated wafer on $\mathrm{Ag}(111)$ surfaces [7-9]. Its properties resemble those of graphene in many ways; for example, silicene exhibits a similarly high charge-carrier mobility [10] and a Dirac-cone-shaped lowenergy band structure at the Brillouin zone corners [11,12]. The aforementioned sublattice buckling, however, introduces interesting new physics into the honeycomb monolayer of silicon. The lack of an in-plane $\sigma_{h}$ mirror symmetry in the crystal, coupled with the intrinsic atomic spin-orbit coupling of $\mathrm{Si}$ atoms, gives rise to a topological band gap on the order of a few meV [13-16]. This band gap can be manipulated by strain [17-20], and also by an external electric field [14] which, above a critical magnitude of the electric field, induces a quantum phase transition from topological insulator to band insulator phase [14-16].

Silicene samples grown on silver substrates have been successfully used to fabricate field effect transistors that provide good performance at room temperature [21-23]. A more widespread utilization of silicene in nanoelectronics and related fields faces considerable challenges due to limitations in existing sample fabrication methods. The chemical vapor deposition growth of silicene on $\operatorname{Ag}(111)$ surfaces remains the most effective means of silicene production, and since the interaction between $\mathrm{Ag}$ and $\mathrm{Si}$ atoms is strong, usually covalent bonds are formed during the growth process. The strong bonding to the substrate makes it difficult to transfer the sample to other substrates without damaging the lattice. Moreover, silicene undergoes reconstructions on the $\operatorname{Ag}(111)$ surface which may occur in a number of ways depending on its orientation, which results in various hybrid silicenesilver structures dispersed on the sample [24-26], including various structures deposited on $\sqrt{13} \times \sqrt{13}$ and $2 \sqrt{3} \times 2 \sqrt{3}$ supercells of the $\operatorname{Ag}(111)$ surface, and the $3 \times 3$ silicene on $4 \times 4 \operatorname{Ag}(111)$ surface. The lattice constant and the structure of the top layer of these surface reconstructions can be measured accurately by scanning tunneling microscopy (STM) [27]; however, the precise atomic structure of the substrate remains hidden as the STM can only detect the top layer, keeping the silver substrate hidden beneath the Si atoms. In conjunction with computational simulations, STM can be used to attempt identification of various types of reconstructions [6]; however, for more complex reconstructions, the experimentally obtained data often suffers from poor resolution [26] which makes precise identification of the reconstruction through the STM simulations challenging.

Further information can be extracted from the structure by the utilization of angular resolved photoelectron spectroscopy (ARPES), which probes the occupied electron bands. However, ARPES measurements have sparked some debate over the precise nature of the bands in the reconstructed silicene. Early measurements identified Dirac cones in the ARPES spectrum and interpreted them as surface Dirac electrons [28], but later it was shown that these Dirac-cone-like bands in fact arose from the silver surface $[29,30]$. More recent studies suggest that while silicene atop $\operatorname{Ag}(111)$ can exhibit conelike bands, these are not related to the Dirac cones at the $K$ point of freestanding silicene but are features originating from hybridized bands due to the interaction between the silicene and the substrate [31-33].

Moreover, silicene is highly unstable under atmospheric conditions. In order to protect materials that degrade swiftly due to, e.g., oxidation, encapsulation in graphene or hexagonal boron nitride (h-BN) can be applied [34,35]. A downside of encapsulation is that it prohibits STM from probing the materials beneath the encapsulating layers, similar to how silicene hides the features of the underlying silver substrate; the information that can be extracted from STM images on encapsulated structures is limited to the lattice parameter which can be obtained if moiré patterns manifest [36-40].

On the other hand, in particular h-BN encapsulation is advantageous in that $\mathrm{h}-\mathrm{BN}$ is transparent and does not prohibit optical spectroscopy. This opens the possibility to utilize Raman spectroscopy to identify the reconstruction even after 

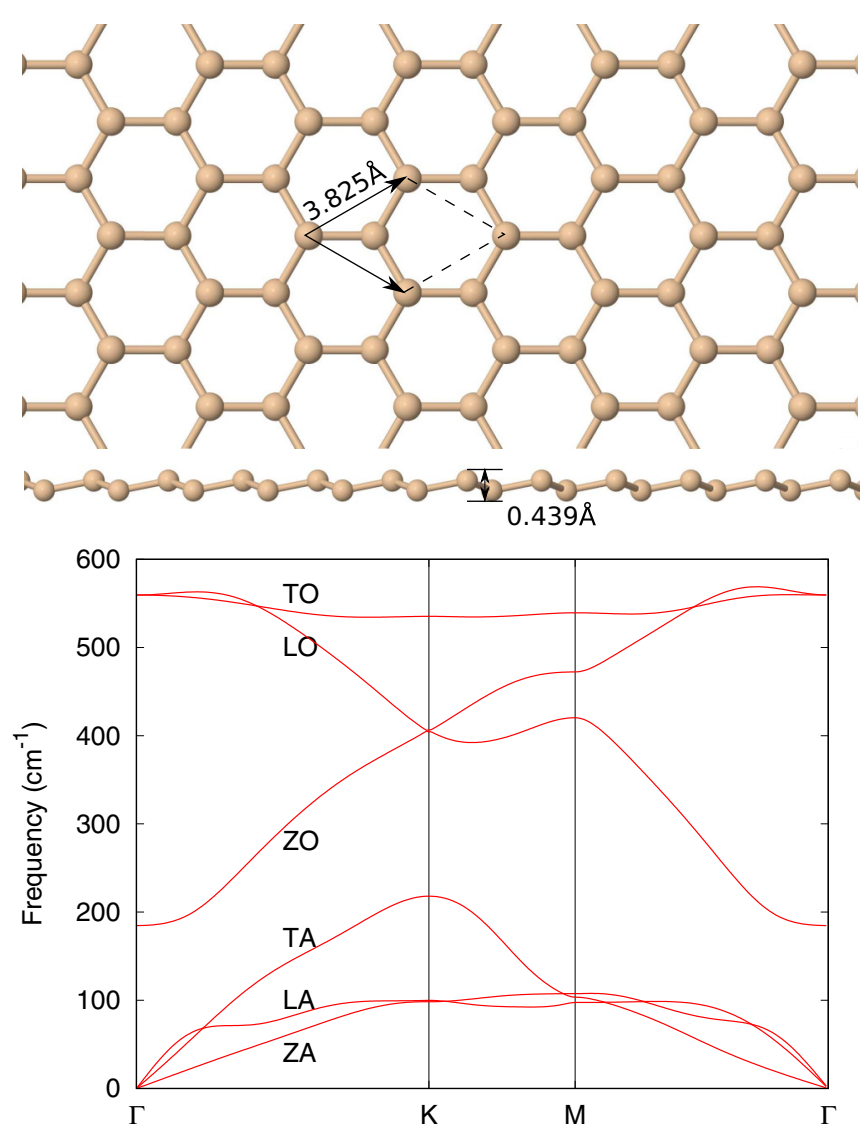

FIG. 1. Lattice structure of silicene in top view and side view (top panel) and the phonon dispersion along the high-symmetry lines (bottom panel). The LDA values [14] for the in-plane lattice constant and the vertical sublattice buckling are displayed.

silicene has been encapsulated in h-BN, thus enabling the detailed spectroscopic study of silicene superstructures in ambient conditions using optical spectroscopy methods.

Raman spectroscopy offers a powerful way to analyze silicene and its reconstructions. This nondestructive technique relies on inelastic scattering of light to probe the phononic excitations in the lattice, and provides this information for both the silicene sheet and the substrate underneath. For the study of silicene grown on specifically silver substrates, Raman spectroscopy can also take advantage of surface enhancement effects [41]. It is also well suited to probe reconstructed surfaces, as reconstructions inevitably bring with them changes to the phonon frequencies and eigenvectors, which can often lead to a vibrational spectrum that can be characteristic of a specific type of reconstruction. Recent experiments found several differences between the Raman spectra of different surface reconstructions of silicene [42-47]. However, the measured Raman spectrum by itself cannot reveal the lattice structure; it is necessary to obtain a point of reference which can be used to assign a Raman signature to a specific reconstruction. Such a point of reference can be provided by first-principles theory. Density functional theory (DFT) is able to compute the fully relaxed atomic structure of a reconstructed silicene on a substrate, and subsequently the corresponding Raman spectrum. Calculating the Raman spectrum of several different surface reconstructions of silicene on $\mathrm{Ag}(111)$ would enable the identification of the reconstructions in the experimental samples by comparing the measured and calculated Raman spectra.

In this work we use DFT in the local density approximation (LDA) to demonstrate the differences between different surface reconstructions of silicene atop $\operatorname{Ag}(111)$. We model the Raman cross section of the reconstructed lattices using the Placzek approximation and calculate the first-order Raman spectra. We compare the results to experiments and suggest a guideline for the classification of measured spectra and the identification of the superstructures.

\section{RESULTS}

To model reconstructions of silicene $\left(\mathrm{Si}_{2}\right)$ on the $\mathrm{Ag}(111)$ surface we use the supercell method. We construct a periodic $\mathrm{Si} / \mathrm{Ag}$ superstructure composed of a supercell of silicene and a supercell of a slab of $\mathrm{Ag}(111)$ where we fix the in-plane lattice parameter of $\operatorname{Ag}(111)$ at that of the bulk crystal and stretch or compress the lattice parameter of silicene in order to achieve commensurability in the structure. We model the $\mathrm{Ag}(111)$ surface by taking seven layers of $\mathrm{Ag}$ atoms in the slab and, to account for surface relaxation effects [48-50], we perform a full structural relaxation on the $\mathrm{Si} / \mathrm{Ag}$ superstructures by keeping the lattice vectors and the atoms in the bottom two layers fixed. This process models the reconstruction of silicene on a macroscopic Ag substrate where the deposited monolayer of $\mathrm{Si}$ atoms is expected to adjust to the substrate.

Due to the mismatch between the lattice constants of silicene and the $\operatorname{Ag}(111)$ surface, strain is introduced into the silicene layer as it is deposited on the surface. Reconstruction occurs as a result of the competition between, on the one hand, the silicene preferring to relax into a minimum strain configuration, and, on the other hand, the strong bonding between $\mathrm{Si}$ and $\mathrm{Ag}$ atoms which promotes crystalline order in the superstructure. In this paper, we carry out first-rinciples modeling of eight possible types of reconstructions, denoted here as structures $\mathrm{A}-\mathrm{H}$, some of which have been observed in experiments [24-26] while others have been predicted theoretically [49]. Details of the computations are provided in the Appendix.

The outcome of the structural relaxation of the $\mathrm{Si} / \mathrm{Ag}$ superstructures is illustrated in Figs. 2(a) and 2(b). The corresponding binding energies $\left(E_{B}\right)$ are listed in Table I, where $E_{B}$ is defined as

$$
E_{B}=\left(E_{\mathrm{Si} / \mathrm{Ag}}-E_{\mathrm{Si}}-E_{\mathrm{Ag}}\right) / N_{\mathrm{Si}}
$$

where $E_{\mathrm{Si} / \mathrm{Ag}}$ is the total energy of the $\mathrm{Si} / \mathrm{Ag}$ superstructure, $E_{\mathrm{Si}}$ and $E_{\mathrm{Ag}}$ are the total energy of the freestanding silicene supercell and the freestanding $\mathrm{Ag}(111)$ slab, respectively, and $N_{\mathrm{Si}}$ is the number of $\mathrm{Si}$ atoms in the superstructure.

We find that the honeycomb structure is well preserved for the five structures $(A-E)$ with relatively small $(<5 \%)$ strain and except for the D structure, the strain and the binding energy is inversely proportional. This relation between the applied strain and the binding energy is not surprising as compressing a lattice will increase its energy, while the energy gain from the $\mathrm{Ag}-\mathrm{Si}$ binding is rather unchanged, thus the superstructure 
(a)

$\mathrm{A}: \sqrt{7} \times \sqrt{7}-\mathrm{I} \mathrm{Si}_{2}$ on $\sqrt{13} \times \sqrt{13} \mathrm{Ag}(111)$

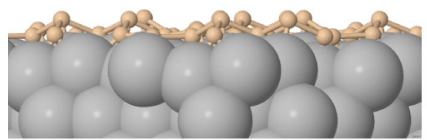

$\mathrm{B}: \sqrt{7} \times \sqrt{7}-\mathrm{II} \mathrm{Si} i_{2}$ on $\sqrt{13} \times \sqrt{13} \mathrm{Ag}(111)$

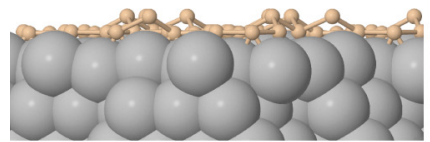

C: $3 \times 3 \mathrm{Si}_{2}$ on $4 \times 4 \mathrm{Ag}(111)$

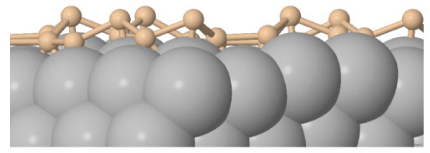

$\mathrm{D}: 2 \times 2 \mathrm{Si}_{2}$ on $\sqrt{7} \times \sqrt{7} \mathrm{Ag}(111)$

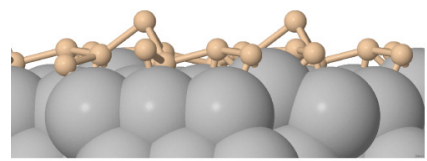

$\mathrm{E}: \sqrt{7} \times \sqrt{7} \mathrm{Si}_{2}$ on $2 \sqrt{3} \times 2 \sqrt{3} \mathrm{Ag}(111)$

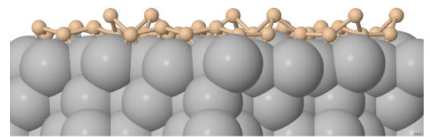

$\mathrm{F}: 3 \times 3 \mathrm{Si}_{2}$ on $\sqrt{13} \times \sqrt{13} \mathrm{Ag}(111)$

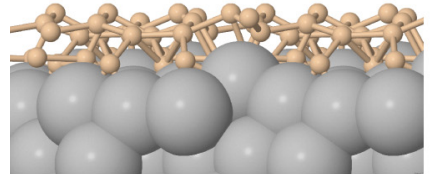

$\mathrm{G}: 2 \sqrt{3} \times 2 \sqrt{3} \mathrm{Si}_{2}$ on $4 \times 4 \mathrm{Ag}(111)$

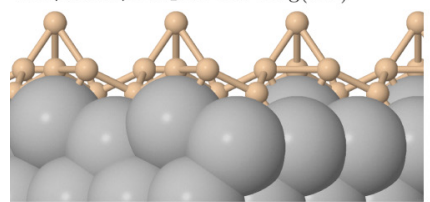

$\mathrm{H}: 2 \sqrt{3} \times 2 \sqrt{3} \mathrm{Si}_{2}$ on $\sqrt{13} \times \sqrt{13} \mathrm{Ag}(111)$

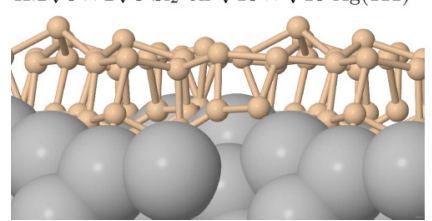

(b)
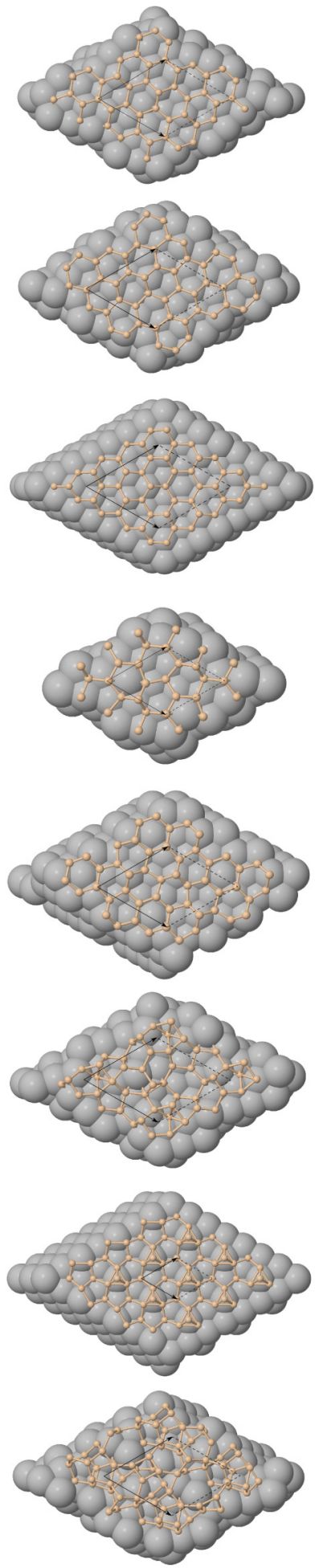

(c)
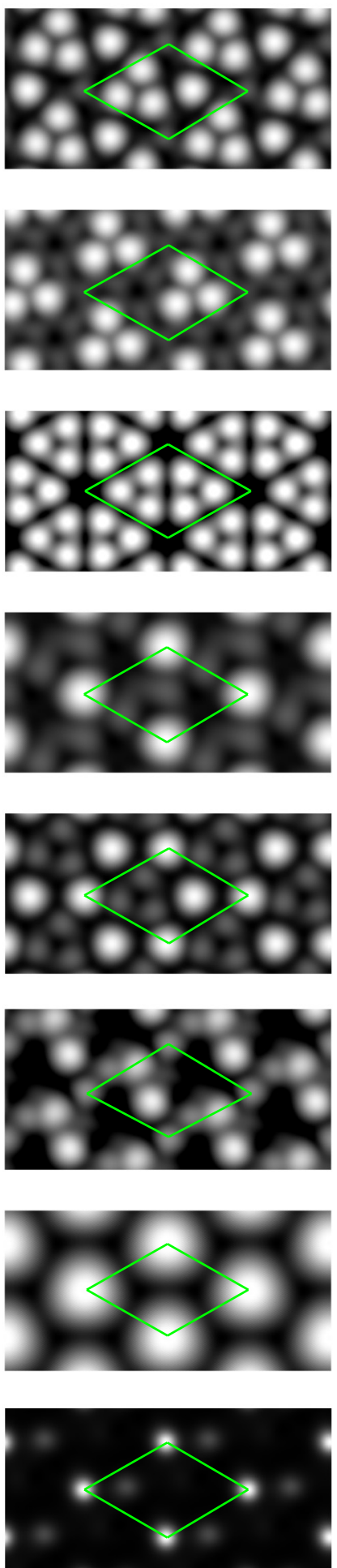

(d)
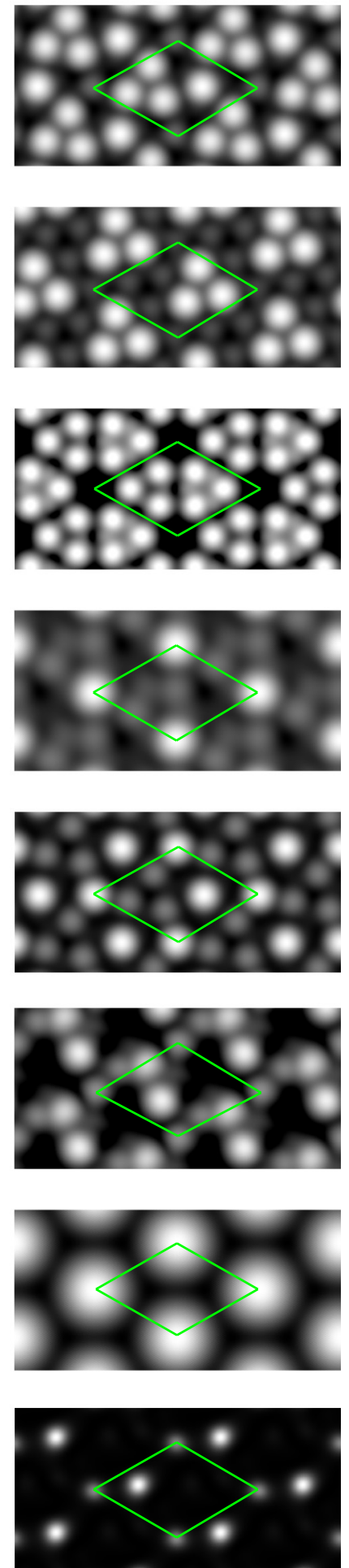

FIG. 2. Atomic structure in side (a) and top (b) view, and STM images at low (c) and high (d) bias for $\mathrm{Si}_{2}$ on $\mathrm{Ag}(111)$ in various reconstructions according to DFT calculations. Bias voltage in the STM simulation was set to $U_{\text {bias }}=-50 \mathrm{mV}$ and $U_{\text {bias }}=-1.4 \mathrm{~V}$ for the low and high bias, respectively.

is expected to be less energetically favorable if the strain is larger.

In contrast, within the subset of the three superstructures where the silicene sheet is heavily distorted $(\mathrm{F}-\mathrm{H})$, the magnitude of the binding energy is proportional to the strain, implying that the structures become more energetically favorable with increasing strain. This perhaps counterintuitive finding can be understood by considering that silicene is a 
TABLE I. Binding energy $\left(E_{B}\right)$ of silicon atoms on the $\operatorname{Ag}(111)$ surface and the corresponding strain on the silicene sheet in various reconstructions.

\begin{tabular}{lcc}
\hline \hline Structure & $E_{B}(\mathrm{meV})$ & Strain $(\%)$ \\
\hline $\mathrm{A}: \sqrt{7} \times \sqrt{7}-\mathrm{I} \mathrm{Si}_{2}$ on $\sqrt{13} \times \sqrt{13} \mathrm{Ag}(111)$ & -459 & -0.7 \\
$\mathrm{~B}: \sqrt{7} \times \sqrt{7}-\mathrm{II} \mathrm{Si}{ }_{2}$ on $\sqrt{13} \times \sqrt{13} \mathrm{Ag}(111)$ & -458 & -0.7 \\
$\mathrm{C}: 3 \times 3 \mathrm{Si}_{2}$ on $4 \times 4 \mathrm{Ag}(111)$ & -436 & -2.8 \\
$\mathrm{D}: 2 \times 2 \mathrm{Si}_{2}$ on $\sqrt{7} \times \sqrt{7} \mathrm{Ag}(111)$ & -152 & -3.6 \\
$\mathrm{E}: \sqrt{7} \times \sqrt{7} \mathrm{Si}_{2}$ on $2 \sqrt{3} \times 2 \sqrt{3} \mathrm{Ag}(111)$ & -419 & -4.6 \\
$\mathrm{~F}: 3 \times 3 \mathrm{Si}_{2}$ on $\sqrt{13} \times \sqrt{13} \mathrm{Ag}(111)$ & -231 & -12.3 \\
$\mathrm{G}: 2 \sqrt{3} \times 2 \sqrt{3} \mathrm{Si}_{2}$ on $4 \times 4 \mathrm{Ag}(111)$ & -252 & -15.7 \\
$\mathrm{H}: 2 \sqrt{3} \times 2 \sqrt{3} \mathrm{Si}_{2}$ on $\sqrt{13} \times \sqrt{13} \mathrm{Ag}(111)$ & -279 & -24.1 \\
\hline \hline
\end{tabular}

metastable phase of silicon. The total energy per Si atom of the bulk three-dimensional silicon crystal is approximately $643 \mathrm{meV}$ below that of silicene according to the LDA; this is due to silicon favoring the $s p^{3}$ hybridization found in the face-centered-cubic lattice over the nearly $s p^{2}$ hybridization found in silicene. Therefore, if silicene is subjected to a sufficiently large distortion caused by compressive strain, the lattice will be stabilized by greater strain, because the compression pushes the bonds closer to $s p^{3}$ hybrids. This is the reason why increasing strain makes the highly distorted superstructures more energetically stable, but one should note that the superstructure where the silicene remains undistorted (and is subjected to very little strain) remains far more stable.

The surface reconstructions studied here, with the exception of structure D which appears to be the least stable of them all, can be separated into two categories based on their binding energies. The members of each group exhibit binding energies that agree within $50 \mathrm{meV}$, despite having clearly distinguishable atomic structures. This agrees with the experimental observation [24-26,46] that structures A, C, and E can grow at the same temperature. The fact that several structures can be grown in given environmental conditions underlines the importance of reliably telling these structures apart in the experiments.

Options for characterizing the reconstructions include scanning imaging methods such as STM [27,51,52]. We simulated the STM images of the reconstructions based on the TersoffHamann approach [53] under high and low bias voltages of $U_{\text {bias }}=-1.4 \mathrm{~V}$ and $U_{\text {bias }}=-50 \mathrm{mV}$, respectively. Images in Figs. 2(c) and 2(d) were calculated by averaging the surface density of states for all electronic states that fell within a $200 \mathrm{meV}$ window centered around the energy level set by the bias voltage. The STM image of the barely strained structures presented in the first five rows of Figs. 2(c) and 2(d) exhibits similar features observed in several experimental $[6,26,54]$ and theoretical $[26,49,55]$ studies. However, our calculated STM image of structure B deviates from the theoretical prediction in Ref. [49] as instead of a single intensive point, a trigonal feature can be seen similar to the STM image of structure A. The difference can be attributed to that this structure appears to be unstable according to the findings in Ref. [49], which implies that there can be considerable variation in the outcome of a structural relaxation for this phase. Similar STM images for the bottom three strained structures in Figs. 2(c) and 2(d) can be found in the literature $[8,24,49]$, and these exhibit similar features to those found in our calculations, albeit, as discussed above, exact identification of these is challenging due to much of the detail in the measurements being blurred. Measuring the STM image with different voltages might help the identification; however, comparing Figs. 2(c) and Fig. 2(d) shows little to no difference between the structures. It is important to realize that STM cannot probe the substrate beneath the silicene and therefore cannot provide a complete description of the reconstruction. In contrast, differences in the precise structure of the reconstructions may give rise to measurable differences in their Raman spectra, which, as we argue below, can be used to identify the structures.

The calculated Raman spectra of the superstructuresbased on the Placzek approximation (see the Appendix for details)_are presented in Fig. 3. For comparison, we highlight the $\Gamma$-point vibrational frequencies of the undistorted silicene layers with vertical lines, where the sole Raman-active mode is plotted with solid, and all other modes with dashed lines; these are obtained from the phonon dispersion shown in Fig. 1 by zone folding them into the Brillouin zone of the corresponding supercell noted over each spectra. For easier comparison of the Raman spectra and the structures, the STM image of each supercell is shown in the inset, calculated for $U_{\text {bias }}=-1.4 \mathrm{~V}$.

The Raman spectra of the superstructures are dominated by the very strong peak originated from the Raman-active peak of the freestanding silicene layer. Due to the strain and the binding with the substrate, the position of this peak substantially changes between different reconstructions. This could, in principle, enable an easy way to distinguish the structures by measuring this Raman mode; however, broadening effects may make it difficult to resolve the fine structure of the Raman spectrum, preventing the identification of the reconstruction from just this mode alone.

The rest of the Raman bands hold information thanks to the varying size of the reconstruction. In the most naive approximation the phonon frequencies in the superstructure would correspond to the phonons of freestanding silicene which zone fold into the $\Gamma$ point of the supercell (illustrated by the dashed vertical lines), but none of them would contribute to the spectrum since they are all inactive in leading-order Raman processes. After reconstruction, however, the actual vibrational modes change, both in terms of frequency and atomic motion, and gain finite Raman intensity. The details of this effect depend on both the size of the silicene supercell and the substrate supercell, which gives rise to measurable differences between the reconstructions in Fig. 3.

Experimentally the most investigated system is the $\mathrm{C}$ structure. Its Raman spectrum is characterized by two major features: the main peak at $514 \mathrm{~cm}^{-1}$ with a broad shoulder and two distinguishable peaks $[46,47,56]$ at 214 and $170 \mathrm{~cm}^{-1}$. Although the first feature is reproduced fairly well in our results in Fig. 3, the second feature is missing, and another double peak is present around $300 \mathrm{~cm}^{-1}$ originated from $\mathrm{ZO}$ phonons [56]. Contradictory experimental data can also be found in the literature, as Ref. [43] reports a wide band around $300 \mathrm{~cm}^{-1}$, while Raman spectra in Refs. [46,47,56] do not show such a peak at all. The absence of significant Raman response at $\propto 200 \mathrm{~cm}^{-1}$ in our calculations can be explained by considering the likely origin of this band in the experiments. The $\mathrm{ZO}$ 

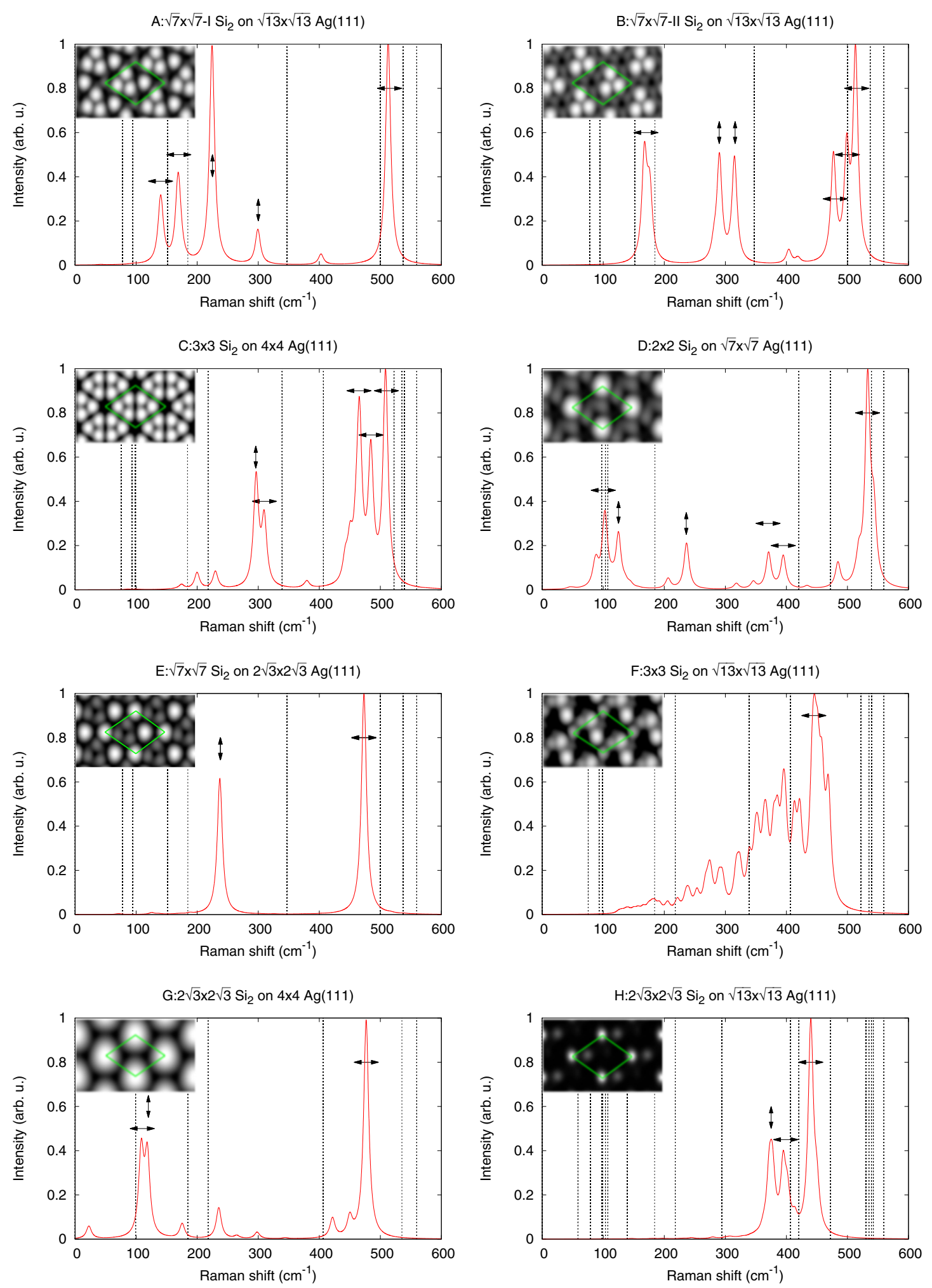

FIG. 3. Calculated Raman spectra of the Si/Ag superstructures, plotted in red solid lines. Vertical lines indicate the frequencies of the freestanding silicene supercells, plotted in dashed lines for the inactive modes and in solid for the only Raman-active mode; insets show calculated STM images for $U_{\text {bias }}=-1.4 \mathrm{~V}$. The dominant directions (in-plane or out-of-plane) of the atomic motion in the vibrations that give rise to the main peaks in the spectra are indicated by arrows.

phonon branch of silicene starts just below $200 \mathrm{~cm}^{-1}$ and is relatively flat near the zone center [56], meaning, that phonons of nonzero wave vector could enhance the Raman response in this frequency region. However, phonons not originating from the $\Gamma$ point can only be excited via higher order processes, such as those induced by defect scattering. The uneven growth within 
the samples certainly promotes defect-induced scattering, and the strong variation in the relative intensity ratio of the two peaks in different measurements supports this interpretation as well. Moreover, the huge tip enhancement in Ref. [56] indicates defect-induced origin, as defect-induced peaks are usually more sensitive to tip enhancement [57]. Since in our calculations we only considered $\Gamma$-point phonons, our spectra do not account for defect-induced processes, which explains the low intensity of the Raman band at $\propto 200 \mathrm{~cm}^{-1}$ in our calculations for structure $\mathrm{C}$.

Comparing the Raman spectra of the other structures to experiments is challenging as the usual notation in the literature only includes the reconstruction of the silver substrate. As discussed before, a given $\mathrm{Ag}(111)$ surface can host several different silicene reconstructions [e.g., the $\sqrt{13} \times \sqrt{13} \mathrm{Ag}(111)$ surface can host four different reconstructions of the silicene lattice], and the reconstructions can have different Raman fingerprints as shown in Fig. 3. These unique fingerprints can be used to identify the silicene structures; for example, in Refs. $[43,44,56]$ the measured Raman spectra of unknown silicene structures deposited on the $\sqrt{13} \times \sqrt{13} \operatorname{Ag}(111)$ surface are reported. In Refs. [43,56] a distinct peak around $240 \mathrm{~cm}^{-1}$ is visible, similar to the Raman spectra of structure A shown in Fig. 3, while the Raman spectra presented in Ref. [44] do not show this feature, although a wide shoulder can be seen, similar to the spectra of structure H. Several experiments $[21,46,56]$ show similar Raman spectra of silicene on the $2 \sqrt{3} \times 2 \sqrt{3} \operatorname{Ag}(111)$ surface, where usually two peaks are visible, much like in our calculated spectra of structure $E$ shown in Fig. 3.

Therefore we can derive simple rules of thumb to distinguish between silicene structures placed on a given silver substrate, as follows.

Silicene placed on the $\sqrt{13} \times \sqrt{13} \operatorname{Ag}(111)$ can be distinguished by two features: the shoulder of the main peak at around $400-500 \mathrm{~cm}^{-1}$, and the low-frequency band at around $200 \mathrm{~cm}^{-1}$. If only the shoulder is present, then the silicene structure can be identified as the $\mathrm{F}$ or the $\mathrm{H}$ structure, depending on whether the shoulder is broad $(\mathrm{F})$ or narrow $(\mathrm{H})$. If the shoulder is absent but the low-frequency band can be seen, that is indicative of the A structure. And although the B structure is considered unstable [49], its fingerprint can in principle be identified with a small shoulder of the main peak with an additional single and a double peak at around 180 and $300 \mathrm{~cm}^{-1}$, respectively.

The two silicene reconstructions atop the $4 \times 4 \mathrm{Ag}(111)$ substrate (structures $\mathrm{C}$ and $\mathrm{G}$ ) can be distinguished by the position of the secondary peak, and also by the intensity of the shoulder of the main peak.

The Raman fingerprint of structures D and E, in which both the silicene and the substrate are different, also exhibits features useful for identification. The spectrum of structure $\mathrm{E}$ contains only one additional peak apart from the main peak, while the spectrum of structure D exhibits numerous extra features, including one at $\propto 100 \mathrm{~cm}^{-1}$.

An important message of our analysis of the Raman response of reconstructed silicene atop $\operatorname{Ag}(111)$ is that Raman spectroscopy enables the identification of the reconstructions, in part through the rich features that appear in the lowfrequency regime of several reconstructions. This regime is accessible by techniques such as those employed in the measurements of the sheer mode in few-layer graphene [58,59] and transition-metal dichalcogenides [60]. Our calculations predict that future experimental studies into the broader range Raman spectroscopy of silicene on $\operatorname{Ag}(111)$ substrates will unveil previously unseen features that will be useful for characterization.

\section{CONCLUSIONS}

We have calculated the reconstructed structures of silicene deposited on $\operatorname{Ag}(111)$ in eight different configurations and modeled their Raman spectra using density functional theory and the Placzek approximation. We have shown that energetically the eight reconstructions are comparable, but their structure and vibrational modes are very different, leading to unique Raman responses. We have demonstrated that Raman spectroscopy can be used to identify each structure and classified a set of distinctive features in the spectra to use as points of reference for the identification. This approach will prove especially useful in future measurements on silicene encapsulated beneath protective layers such as hexagonal boron nitride.

\section{ACKNOWLEDGMENTS}

Support from the Hungarian National Research, Development and Innovation Office (NKFIH, Grants No. K-115608 and No. K-108676) is acknowledged. We acknowledge NIIF for awarding us access to a resource based in Hungary at Debrecen. This research was supported by the National Research Development and Innovation Office of Hungary within the Quantum Technology National Excellence Program (Project No. 2017-1.2.1-NKP-2017-00001) G.K. acknowledges support from the New National Excellence Program (UNKP) of the Ministry of Human Capacities in Hungary. V.Z. acknowledges support from the Graphene Flagship Project, the N8 Polaris service, the ARCHER National UK Supercomputer (RAP Project e547), and the Computational Shared Facility at the University of Manchester. J.K. acknowledges the Bolyai program of the Hungarian Academy of Sciences.

\section{APPENDIX: METHODS}

The superstructures in this work are modeled using firstprinciples density functional theory and are carried out with the VASP $[61,62]$ code, using the projector-augmented wave method within the framework of the LDA with a plane-wave basis with a cutoff energy of $500 \mathrm{eV}$. As we model surfaces with different in-plane supercell lattice constants-as defined by the $\operatorname{Ag}(111)$ slabs-we employ a $6 \times 6 \times 1$ k-point $\Gamma$-centered Monkhorst-Pack grid [63] for the $4 \times 4 \mathrm{Ag}(111)$ and a $7 \times$ $7 \times 1$ k-point grid for the $\sqrt{13} \times \sqrt{13}$ and the $2 \sqrt{3} \times 2 \sqrt{3}$ $\operatorname{Ag}(111)$ throughout the calculations, which ensures that the Brillouin zone is sampled with approximately the same density as the $24 \times 24 \times 1$ Monkhorst-Pack grid in the primitive cell of a slab of $\operatorname{Ag}(111)$ (corresponding to $\approx 0.07 \AA^{-2}$ area per $\mathbf{k}$ point in the Brillouin zone).

Phonon calculations are carried out using the frozen phonon approximation. The dynamical matrix is calculated by 


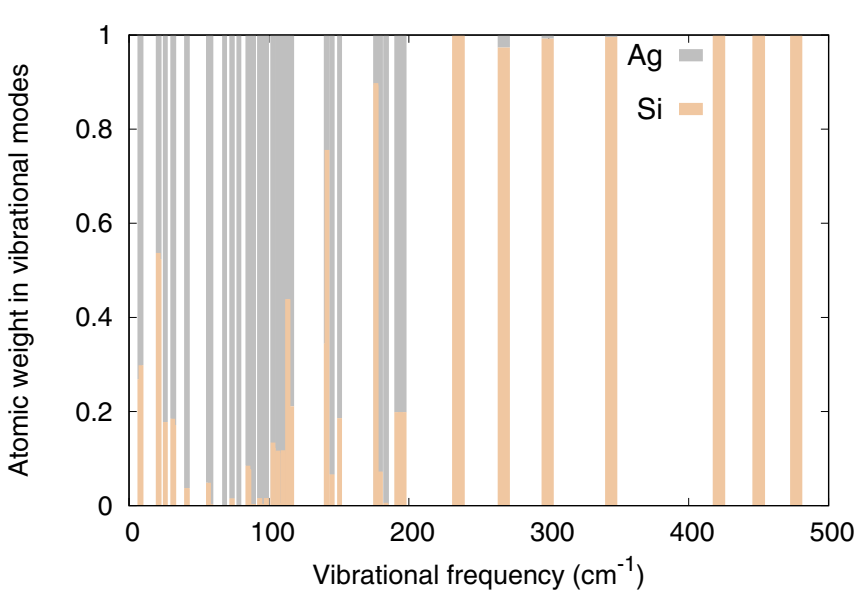

FIG. 4. Normalized relative atomic weights in the $\Gamma$-point phonons in the superstructure comprising $\sqrt{3} \times \sqrt{3} \mathrm{Si}_{2}$ on $2 \times 2$ $\operatorname{Ag}(111)$.

computing the second derivatives of the total energy with respect to atomic displacements, where the atoms are displaced by $0.015 \AA$. To calculate the whole dynamical matrix of the superstructure, all $\mathrm{Si}$ and and $\mathrm{Ag}$ atoms should be displaced; however, if the phonons of the silicene and the $\operatorname{Ag}(111)$ slab are sufficiently localized on the corresponding subsystem, then the correct frequencies and eigenvectors of silicene vibrations can be calculated by displacing only the $\mathrm{Si}$ atoms. To test that the frequencies can be accurately obtained this way, we calculate the $\Gamma$-point phonon frequencies by displacing all atoms on the example of the $\sqrt{3} \times \sqrt{3} \mathrm{Si}_{2}$ on the $2 \times 2 \mathrm{Ag}(111)$ superstructure.

The contribution of the $\mathrm{Si}$ and $\mathrm{Ag}$ atoms to a given phonon mode is calculated by summing the amplitudes of the normalized atomic movements for each type of atoms. In Fig. 4 we show the weights of the atoms in each mode as a function of the phonon frequencies at the $\Gamma$ point. It can be clearly seen that above a frequency of $\approx 200 \mathrm{~cm}^{-1}$ the vibrations of the silicene layer are nearly completely decoupled from the $\operatorname{Ag}(111)$ slab beneath. At low frequencies some of the $\mathrm{Si}$ and $\mathrm{Ag}$ vibrations are mixed; however, when we calculate the Raman intensity as described below, we find that these modes are Raman inactive, therefore we conclude that it is a valid approximation to only displace the silicon atoms during the phonon calculations.

To compute the Raman intensities we utilize the Placzek approximation [64]. The Raman spectra are obtained by calculating the first derivative of the linear polarizability tensor with respect to the phonon normal modes,

$$
I\left(\omega, \omega_{l}\right)=\sum_{\nu} \frac{\omega_{s}^{4}}{\omega_{\nu}} \sum_{\rho, \sigma}\left|\frac{\partial \alpha_{\rho \sigma}\left(\omega_{l}\right)}{\partial Q_{\nu}}\right|^{2} \delta\left(\omega-\omega_{\nu}\right)\left[n\left(\omega_{\nu}\right)+1\right],
$$

where $\omega_{l}, \omega_{s}, \omega_{v}$ are the frequencies of the exciting laser, the scattered light, and the vibrational modes, respectively, $\alpha_{\rho \sigma}$ is the polarizability tensor, $Q_{\nu}$ are the normal modes, $\delta(x)$ is a normalized Lorentzian function with a full width at half maximum value of $10 \mathrm{~cm}^{-1}$ and $n\left(\omega_{v}\right)$ is the Bose-Einstein distribution at room temperature.

Usual exerimental setups utilize backscattering geometry, thus the polarization of the incident and scattered light are parallel to the surface. In order to compare our results with the previously found experimental works, the average conducted in Eq. (A1) was made over the $x x, x y, y x$, and $y y$ components of the polarizability tensor.

In the literature the intensities are often calculated by taking the derivatives of the static polarizability $\alpha_{\rho, \sigma}$; however, we found that the static polarizability (and its derivative) diverges in gapless systems such as silicene or the $\mathrm{Ag}(111)$ substrate and therefore cannot be used to predict the Raman intensities of the structures studied here. To avoid this problematic behavior of the static polarizability we used the first derivative of the frequency-dependent polarizability tensor evaluated at a finite laser energy of $2.33 \mathrm{eV}$ to calculate the Raman intensities.

The frequency-dependent polarizability tensor is calculated from first principles via linear response as implemented in VASP [65], which computes the frequency-dependent dielectric function $\epsilon(\omega)$, allowing us to obtain the polarizability as $\alpha(\omega)=\epsilon(\omega)-1$. To reach convergence in the dielectric function it is necessary to take a large number of empty bands into account, which we achieve by including all empty states up to $50 \mathrm{eV}$ above the Fermi level. To obtain the Raman intensities, we calculate the polarizability after displacing the atoms along the phonon normal modes with a displacement amplitude of $0.015 \AA$, and calculate its numerical derivative.
[1] K. S. Novoselov, A. K. Geim, S. V. Morozov, D. Jiang, Y. Zhang, S. V. Dubonos, I. V. Grigorieva, and A. A. Firsov, Science 306, 666 (2004).

[2] K. Takeda and K. Shiraishi, Phys. Rev. B 50, 14916 (1994).

[3] B. Aufray, A. Kara, S. Vizzini, H. Oughaddou, C. Léandri, B. Ealet, and G. Le Lay, Appl. Phys. Lett. 96, 183102 (2010).

[4] P. De Padova, C. Quaresima, C. Ottaviani, P. M. Sheverdyaeva, P. Moras, C. Carbone, D. Topwal, B. Olivieri, A. Kara, H. Oughaddou, B. Aufray, and G. Le Lay, Appl. Phys. Lett. 96, 261905 (2010).

[5] B. Lalmi, H. Oughaddou, H. Enriquez, A. Kara, S. Vizzini, B. Ealet, and B. Aufray, Appl. Phys. Lett. 97, 223109 (2010).
[6] P. Vogt, P. De Padova, C. Quaresima, J. Avila, E. Frantzeskakis, M. C. Asensio, A. Resta, B. Ealet, and G. Le Lay, Phys. Rev. Lett. 108, 155501 (2012).

[7] C. Hogan, O. Pulci, P. Gori, F. Bechstedt, D. S. Martin, E. E. Barritt, A. Curcella, G. Prevot, and Y. Borensztein, Phys. Rev. B 97, 195407 (2018).

[8] B. Feng, Z. Ding, S. Meng, Y. Yao, X. He, P. Cheng, L. Chen, and K. Wu, Nano Lett. 12, 3507 (2012).

[9] C.-L. Lin, R. Arafune, K. Kawahara, N. Tsukahara, E. Minamitani, Y. Kim, N. Takagi, and M. Kawai, Appl. Phys. Express 5, 045802 (2012).

[10] Z.-G. Shao, X.-S. Ye, L. Yang, and C.-L. Wang, J. Appl. Phys. 114, 093712 (2013). 
[11] S. Cahangirov, M. Topsakal, E. Aktürk, H. Sahin, and S. Ciraci, Phys. Rev. Lett. 102, 236804 (2009).

[12] E. Cinquanta, E. Scalise, D. Chiappe, C. Grazianetti, B. van den Broek, M. Houssa, M. Fanciulli, and A. Molle, J. Phys. Chem. C 117, 16719 (2013).

[13] C.-C. Liu, H. Jiang, and Y. Yao, Phys. Rev. B 84, 195430 (2011).

[14] N. D. Drummond, V. Zólyomi, and V. I. Fal'ko, Phys. Rev. B 85, 075423 (2012).

[15] T. Kaloni, M. Tahir, and U. Schwingenschlögl, Sci. Rep. 3, 3192 (2013).

[16] M. Ezawa, J. Phys. Soc. Jpn. 84, 121003 (2015).

[17] R. Qin, W. Zhu, Y. Zhang, and X. Deng, Nanoscale Res. Lett. 9, 521 (2014).

[18] S. Phonapha, A. Suwanvarangkoon, and B. Soodchomshom, Phys. Lett. A 381, 2754 (2017).

[19] H. Zhao, Phys. Lett. A 376, 3546 (2012).

[20] J.-A. Yan, S.-P. Gao, R. Stein, and G. Coard, Phys. Rev. B 91, 245403 (2015).

[21] L. Tao, E. Cinquanta, D. Chiappe, C. Grazianetti, M. Fanciulli, M. Dubey, A. Molle, and D. Akinwande, Nat. Nanotechnol. 10, 227 (2015).

[22] N. Pournaghavi, M. Esmaeilzadeh, A. Abrishamifar, and S. Ahmadi, J. Phys.: Condens. Matter 29, 145501 (2017).

[23] G. L. Lay, Nat. Nanotechnol. 10, 202 (2015).

[24] H. Jamgotchian, Y. Colignon, N. Hamzaoui, B. Ealet, J. Y. Hoarau, B. Aufray, and J. P. Bibérian, J. Phys.: Condens. Matter 24, 172001 (2012).

[25] J. Avila, P. D. Padova, S. Cho, I. Colambo, S. Lorcy, C. Quaresima, P. Vogt, A. Resta, G. L. Lay, and M. C. Asensio, J. Phys.: Condens. Matter 25, 262001 (2013).

[26] Z.-L. Liu, M.-X. Wang, J.-P. Xu, J.-F. Ge, G. L. Lay, P. Vogt, D. Qian, Chun-Lei Gao, C. Liu, and J.-F. Jia, New J. Phys. 16, 075006 (2014).

[27] H. Enriquez, S. Vizzini, A. Kara, B. Lalmi, and H. Oughaddou, J. Phys.: Condens. Matter 24, 314211 (2012).

[28] P. De Padova, P. Vogt, A. Resta, J. Avila, I. Colambo, C. Quaresima, C. Ottaviani, B. Olivieri, T. Bruhn, T. Hirahara, T. Shirai, S. Hasegawa, M. Asensio, and G. Le Lay, Appl. Phys. Lett. 102, 163106 (2013).

[29] P. Gori, O. Pulci, F. Ronci, S. Colonna, and F. Bechstedt, J. Appl. Phys. 114, 113710 (2013).

[30] Y.-P. Wang and H.-P. Cheng, Phys. Rev. B 87, 245430 (2013).

[31] Y. Feng, D. Liu, B. Feng, X. Liu, L. Zhao, Z. Xie, Y. Liu, A. Liang, C. Hu, Y. Hu, S. He, G. Liu, J. Zhang, C. Chen, Z. Xu, L. Chen, K. Wu, Y.-T. Liu, H. Lin, Z.-Q. Huang, C.-H. Hsu, F.-C. Chuang, A. Bansil, and X. J. Zhou, Proc. Natl. Acad. Sci. USA 113, 14656 (2016).

[32] C. Lian and S. Meng, Phys. Rev. B 95, 245409 (2017).

[33] S. K. Mahatha, P. Moras, P. M. Sheverdyaeva, V. Bellini, T. O. Menteş, A. Locatelli, R. Flammini, K. Horn, and C. Carbone, J. Electron Spectrosc. Relat. Phenom. 219, 2 (2017).

[34] A. K. Geim and I. V. Grigorieva, Nature (London) 499, 419 (2013).

[35] D. A. Bandurin, A. V. Tyurnina, G. L. Yu, A. Mishchenko, V. Zólyomi, S. V. Morozov, R. K. Kumar, R. V. Gorbachev, Z. R. Kudrynskyi, S. Pezzini, Z. D. Kovalyuk, U. Zeitler, K. S. Novoselov, A. Patanè, L. Eaves, I. V. Grigorieva, V. I.
Fal'ko, A. K. Geim, and Y. Cao, Nat. Nanotechnol. 12, 223 (2017).

[36] J. Xue, J. Sanchez-Yamagishi, D. Bulmash, P. Jacquod, A. Deshpande, K. Watanabe, T. Taniguchi, P. Jarillo-Herrero, and B. J. LeRoy, Nat. Mater. 10, 282 (2011).

[37] J. Park, J. Lee, L. Liu, K. W. Clark, C. Durand, C. Park, B. G. Sumpter, A. P. Baddorf, A. Mohsin, M. Yoon, G. Gu, and A.-P. Li, Nat. Commun. 5, 5403 (2014).

[38] Q. Zhang, Y. Chen, C. Zhang, C.-R. Pan, M.-Y. Chou, C. Zeng, and C.-K. Shih, Nat. Commun. 7, 13843 (2016).

[39] J. Lee, M. Kim, J. R. Chelikowsky, and G. Kim, Phys. Rev. B 94, 035447 (2016).

[40] J.-B. Qiao, Y. Gong, W.-J. Zuo, Y.-C. Wei, D.-L. Ma, H. Yang, N. Yang, K.-Y. Qiao, J.-A. Shi, L. Gu, and L. He, Phys. Rev. B 95, 201403 (2017).

[41] F. Schedin, E. Lidorikis, A. Lombardo, V. G. Kravets, A. K. Geim, A. N. Grigorenko, K. S. Novoselov, and A. C. Ferrari, ACS Nano 4, 5617 (2010).

[42] E. Scalise, M. Houssa, G. Pourtois, B. v. d. Broek, V. Afanas'ev, and A. Stesmans, Nano Res. 6, 19 (2013).

[43] E. Scalise, E. Cinquanta, M. Houssa, B. van den Broek, D. Chiappe, C. Grazianetti, G. Pourtois, B. Ealet, A. Molle, M. Fanciulli, V. V. Afanas'ev, and A. Stesmans, Appl. Surf. Sci. 291, 113 (2014).

[44] J. Zhuang, X. Xu, Y. Du, K. Wu, L. Chen, W. Hao, J. Wang, W. K. Yeoh, X. Wang, and S. X. Dou, Phys. Rev. B 91, 161409 (2015).

[45] A. Díaz Álvarez, T. Zhu, J. P. Nys, M. Berthe, M. Empis, J. Schreiber, B. Grandidier, and T. Xu, Surf. Sci. 653, 92 (2016).

[46] D. Solonenko, O. D. Gordan, G. L. Lay, D. R. T. Zahn, and P. Vogt, Beilstein J. Nanotechnol. 8, 1357 (2017).

[47] D. Solonenko, O. D. Gordan, G. L. Lay, H. Sahin, S. Cahangirov, D. R. T. Zahn, and Patrick Vogt, 2D Mater. 4, 015008 (2017).

[48] V. Zólyomi, L. Vitos, S. K. Kwon, and J. Kollár, J. Phys.: Condens. Matter 21, 095007 (2009).

[49] P. Pflugradt, L. Matthes, and F. Bechstedt, Phys. Rev. B 89, 035403 (2014).

[50] W. Wang, W. Olovsson, and R. I. G. Uhrberg, Phys. Rev. B 92, 205427 (2015).

[51] A. Resta, T. Leoni, C. Barth, A. Ranguis, C. Becker, T. Bruhn, P. Vogt, and G. L. Lay, Sci. Rep. 3, 2399 (2013).

[52] Z. Majzik, M. R. Tchalala, M. Švec, P. Hapala, H. Enriquez, A. Kara, A. J. Mayne, G. Dujardin, P. Jelínek, and H. Oughaddou, J. Phys.: Condens. Matter 25, 225301 (2013).

[53] J. Tersoff and D. R. Hamann, Phys. Rev. B 31, 805 (1985).

[54] D. Chiappe, C. Grazianetti, G. Tallarida, M. Fanciulli, and A. Molle, Adv. Mater. 24, 5088 (2012).

[55] Z. Hong-Xia, Q. Ru-Ge, W. Yang-Yang, S. Jun-Jie, and L. Jing, Chin. Phys. B 24, 087308 (2015).

[56] S. Sheng, J.-b. Wu, X. Cong, W. Li, J. Gou, Q. Zhong, P. Cheng, P.-h. Tan, L. Chen, and K. Wu, Phys. Rev. Lett. 119, 196803 (2017).

[57] R. Beams, L. G. Cançado, A. Jorio, S.-H. Oh, and L. Novotny, Phys. Rev. Lett. 113, 186101 (2014).

[58] P. H. Tan, W. P. Han, W. J. Zhao, Z. H. Wu, K. Chang, H. Wang, Y. F. Wang, N. Bonini, N. Marzari, N. Pugno, G. Savini, A. Lombardo, and A. C. Ferrari, Nat. Mater. 11, 294 (2012).

[59] A. C. Ferrari and D. M. Basko, Nat. Nanotechnol. 8, 235 (2013). 
[60] Y. Zhao, X. Luo, H. Li, J. Zhang, P. T. Araujo, C. K. Gan, J. Wu, H. Zhang, S. Y. Quek, M. S. Dresselhaus, and Q. Xiong, Nano Lett. 13, 1007 (2013).

[61] G. Kresse and J. Hafner, Phys. Rev. B 47, 558 (1993).

[62] G. Kresse and J. Furthmüller, Phys. Rev. B 54, 11169 (1996).
[63] H. J. Monkhorst and J. D. Pack, Phys. Rev. B 13, 5188 (1976).

[64] R. Martin and L. Falicov, Light Scattering in Solids I, Topics in Applied Physics Vol. 8 (Springer, Berlin/Heidelberg, 1983), pp. 79-145.

[65] M. Gajdos, K. Hummer, G. Kresse, J. Furthmüller, and F. Bechstedt, Phys. Rev. B 73, 045112 (2006). 